\title{
Distributed optical fibre sensing with enhanced frequency range and sensitivity for structural health monitoring
}

\author{
Ali Masoudi and Trevor P. Newson \\ Optoelectronics Research Centre (ORC), University of Southampton, SO17 1BJ \\ A.Masoudi@soton.ac.uk
}

\begin{abstract}
A distributed optical fibre sensing system is studied which uses a PZT to modulate the phase of the backscattered Rayleigh light in order to enhance the frequency range and strain sensitivity of the sensing system.

OCIS codes: (060.2370) Fiber optics sensors; (290.5870) Rayleigh scattering.
\end{abstract}

\section{Introduction}

Distributed optical fibre sensors (DOFS) are playing an ever more important role in monitoring the health of large scale infrastructures where employing a network of individual point sensors is impractical. From distributed temperature sensors (DTS) looking for hot spots along transmission lines [1,2] to distributed acoustic sensors (DAS) monitoring geophysical activities in boreholes [3, 4], DOFS are becoming established sensing tools in a wide range of applications capable of mapping physical fields over a vast areas or long distances using a robust, lowprofile, and flexible sensing element.

The initial studies on DOFS were focused on developing systems to measure static or quasi-static phenomena such as fibre loss or temperature distribution along the sensing fibre. In recent years, however, a new class of distributed sensors have been demonstrated capable of measuring dynamic phenomena using different sensing techniques [5]. One of these techniques uses the phase of the Rayleigh backscattered light to measure dynamic changes along the fibre [6-8].

In this report, we built on our previous work to enhance the frequency and sensing range, and strain sensitivity of the sensing system by modulating the backscattered light at the receiving Mach-Zehnder interferometer (MZI). In this technique, a PZT is attached to one of the two arms of the interferometer to modulate the phase of the backscattered light and generate a carrier signal to shift the frequency spectrum with it. Since differentiate and crossmultiplying demodulation technique yields a higher noise level at lower frequencies (figure 1(a)), low frequency strains are harder to detect. By mixing the backscattered light with a carrier and moving the signal to the frequency range where the noise level is lower, the same low frequency strain perturbations can be measured (figure 1(b)).

a)

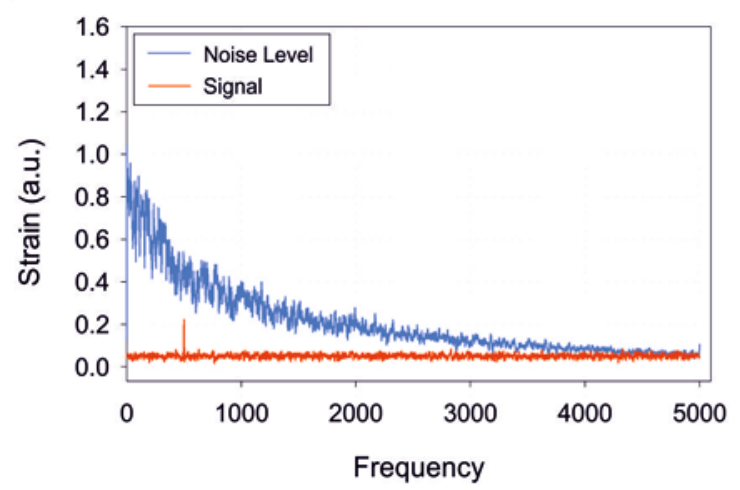

b)

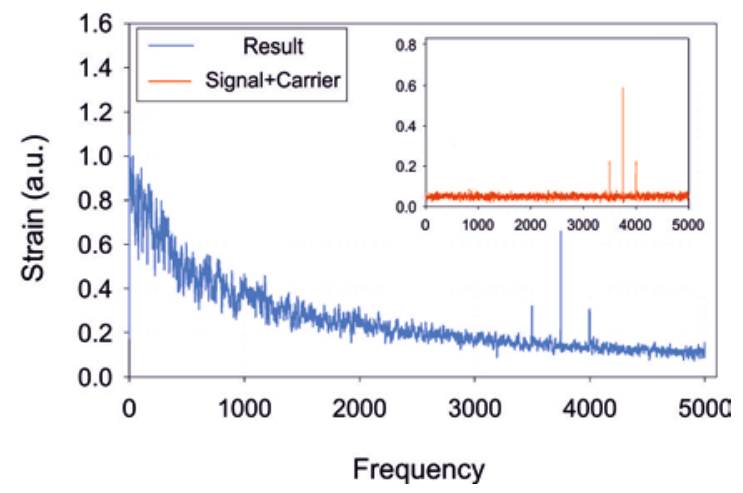

Fig. 1. Principle of low-frequency dynamic strain measurement by mixing the backscattered light with a carrier signal. (a) Signal level below the noise floor of the system prior to modulation, (b) Carrier and signal peaks after mixing backscattered light with carrier signal.

\section{Experimental Setup}

The experimental setup is shown in figure 2. A 1550nm distributed feedback (DFB) laser diode was directly modulated to generate $10 \mathrm{~ns}$ pulse with a peak power of $10 \mathrm{~mW}$ and a repetition rate of $100 \mu \mathrm{s}$. The pulse was amplified to a peak power of $1 \mathrm{~W}$ by an Erbium-doped fibre amplifier (EDFA1). The amplified probe pulse was then 
filtered using a fibre Bragg grating (FBG). In the new experimental setup, a single FBG was used to filter the amplified spontaneous emission (ASE) from the two EDFAs. Using this architecture eliminates the need for two matched-FBGs and allowed the use of a FBG with narrower line-width. To block the ASE from leaking into the three detectors, the output of the EDFA1 was coupled to the FBG filter via an AOM. The AOM was switched on for a short period of time during which the probe pulse was propagating and switched back off until the arrival of the next probe pulse.

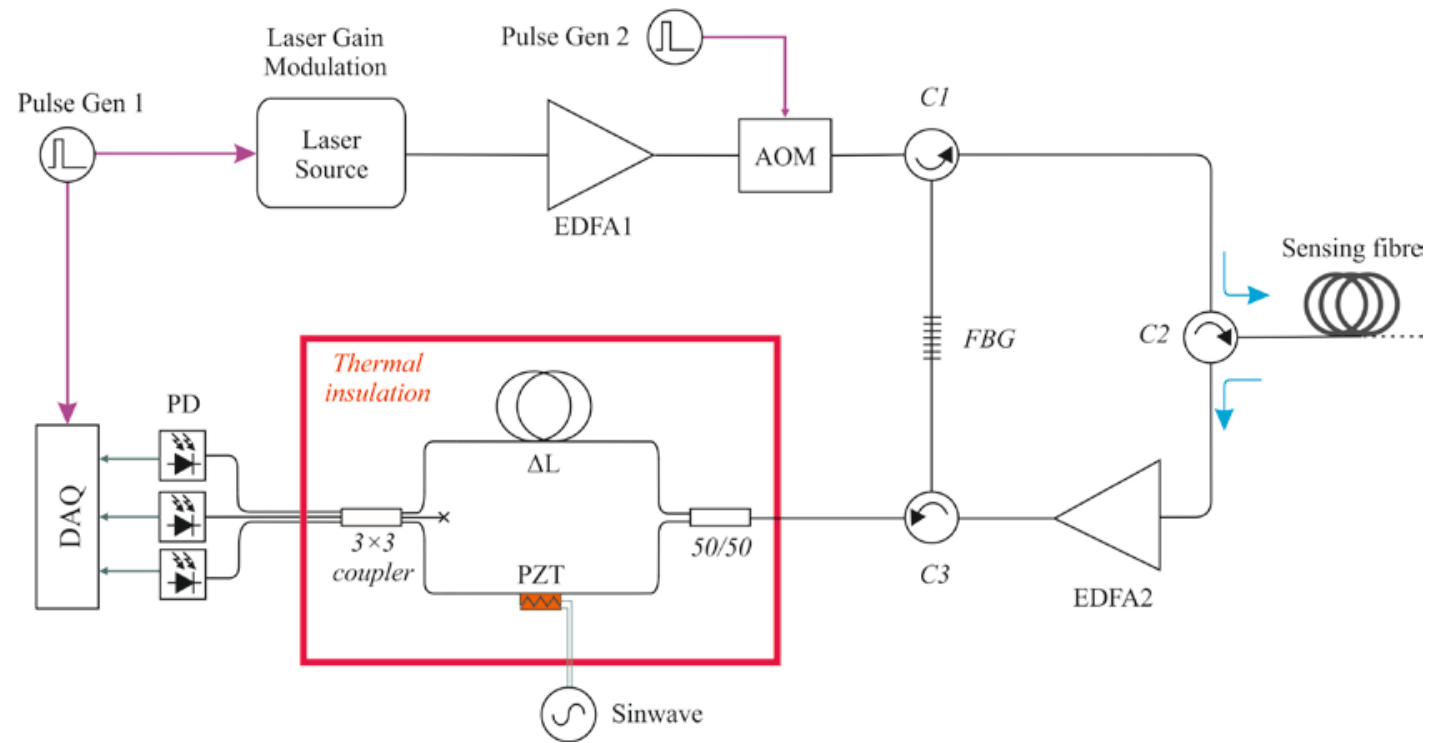

Fig. 2. Experimental Setup. EDFA: Erbium-doped fibre amplifier, AOM: Acousto-optic modulator, C: Circulator, PD: Photodetector, DAQ: Data acquisition unit.

The backscattered light from the sensing fibre was collected via the circulator C2, amplified by the second optical amplifier (EDFA2) and filtered using the same FBG as shown in figure 1. The filtered signal was then fed into the thermally insulated MZI with path-imbalance of 2m using a 50/50 coupler. A PZT was attached to one of the MZI arms to modulate the phase of the backscattered light. Three photoreceivers were used to detect the light from the $3 \times 3$ coupler at the output of the MZI. The output of the photoreceivers were collected using a $250 \mathrm{MHz}$ oscilloscope at a sampling rate of $625 \mathrm{MSa} / \mathrm{s}$.

The acquired signal on each detector consisted of a train of backscattered traces. At the first step of signal processing, the data array from each detector was rearranged to form a matrix in which the matrix columns represented a backscattered trace from the fibre and the matrix rows represented the back scattered light at a particular position along the sensing fibre as a function of time. The three matrices from the output of the three detectors were then fed into a differentiate and cross-multiplying demodulator to extract the phase information of the backscattered light. A fast Fourier transform was performed at each sampled position to identify the frequency components of the dynamic perturbation at each point along the fibre.

The test arrangement was consisted of a 3m of sensing fibre, modulated with a disk PZT, was sandwiched between a $4.5 \mathrm{~km}$ and a $500 \mathrm{~m}$ single mode optical fibres. The delay fibres were thermally and acoustically isolated.

\section{Results and Discussion}

Figure 3 shows the experimental results obtained with the setup shown in figure 1. The 3D diagram of figure 3(a) shows the FFT of the phase-detector output between $4650 \mathrm{~m}$ and $4850 \mathrm{~m}$ where a 200n $\varepsilon$ longitudinal strain with a frequency of $300 \mathrm{~Hz}$ were applied on the fibre. In this experiment, the frequency of the PZT at the receiving interferometer was set to $2000 \mathrm{~Hz}$. Figure 3(b) shows the 2D cross-section of the 3D diagram. In this figure, the $2000 \mathrm{~Hz}$ carrier frequency and its two side-bands at $1707 \mathrm{~Hz}$ and $2301 \mathrm{~Hz}$ are evident. The value of the detected frequencies and strains are within the expected tolerance of the sensor. It can be seen that the two side-bands are $\sim 300 \mathrm{~Hz}$ away from the carrier frequency which is equal to the frequency of the strain applied on the sensing fibre. 

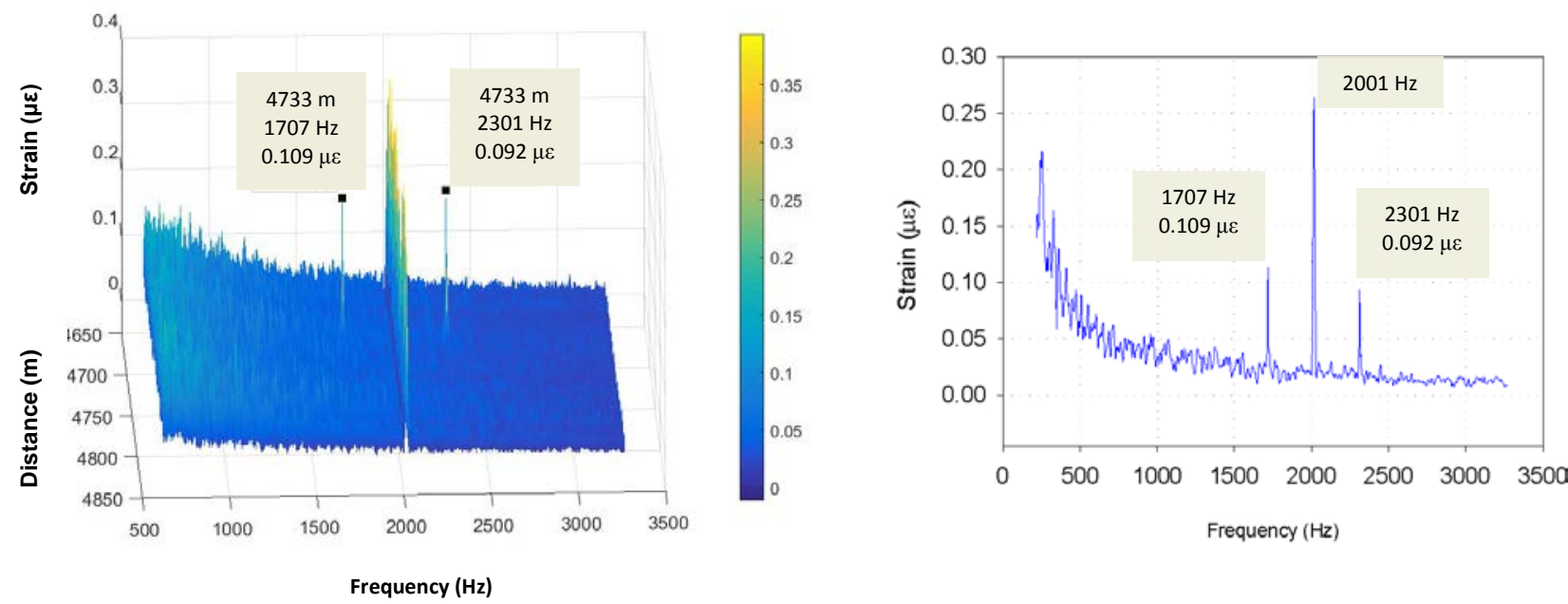

Fig. 3. (a) The FFT of the phase-detector output between $4650 \mathrm{~m}$ and $4850 \mathrm{~m}$ where a $200 \mathrm{n} \varepsilon$ longitudinal strain with a frequency of $300 \mathrm{~Hz}$ were applied on $3 \mathrm{~m}$ of sensing fibre. (b) The $2 \mathrm{D}$ cross-section of the

The experimental result shows that the proposed sensing procedure is capable of measuring strain as low as $100 \mathrm{~Hz}$ and $60 \mathrm{n} \varepsilon$.

It is important to mention that same principle mentioned in the second section can be employed to detect vibrations beyond the Nyquist rate of the distributed sensor. By adjusting the carrier frequency to $18 \mathrm{kHz}$, we have managed to detect a $20 \mathrm{kHz}$ dynamic strain at the disk PZT. The $20 \mathrm{kHz}$ vibration appeared as a $2 \mathrm{kHz}$ peak at the position where the sensing fibre was attached to the disk PZT.

\section{Conclusion}

A distributed optical fibre sensor has been demonstrated with an extended frequency and sensing range. It is shown that by mixing the backscattered light with a carrier signal, low-frequency dynamic perturbations can be frequency-shifted to the part of the frequency spectrum with a lower noise level and, consequently, being measured. It is also shown that the same principle can be used to detect vibrations beyond the Nyquist rate of the sensing system by using high-frequency carrier signal.

\section{References}

[1] G. Yilmaz and S. E. Karlik, "A distributed optical fiber sensor for temperature detection in power cables," Sensor Actuat-A-Phys. 125, 148155 (2006).

[2] A. Ukil, H. Braendle, and P. Krippner, "Distributed temperature sensing: Review of technology and applications,” IEEE Sens. J. 12, 885 - 892 (2011).

[3] T. Daley et al "Field testing of fiber-optic distributed acoustic sensing (DAS) for subsurface seismic monitoring," The Leading Edge 32, 699706 (2013).

[4] A. Mateeva et al. "Distributed acoustic sensing for reservoir monitoring with vertical seismic profiling,” Geophysical Prospecting 62, 679-92 (2014).

[5] A. Masoudi and T. P. Newson, “Contributed Review: Distributed optical fibre dynamic strain sensing,” Rev. Sci. Instrum. 87, 011501 (2016).

[6] A. Masoudi, M. Belal and T. P. Newson, “A distributed optical fibre dynamic strain sensor based on phase-OTDR,” Meas. Sci. Technol. 24, 085204 (2013).

[7] C. Wang et al. "Distributed acoustic mapping based on interferometry of phase optical time-domain reflectometry,” Opt. Commun. 346, 172177 (2015).

[8] G. Tu et al. “The Development of an $\Phi$-OTDR System for Quantitative Vibration Measurement,” IEEE Photon. Technol. Lett. 27, 1349-1352 (2015). 